\title{
Research on Equipment Readiness Assessment Model
}

\author{
Liang Ma \\ Department of Surface Ship Command, Dalian Naval Academy, Dalian, 116018,China \\ 446982051@qq.com
}

\begin{abstract}
Keywords: Equipment combat readiness; Assessment; Model
\end{abstract}
\begin{abstract}
From the view point of man-machine environment system engineering, in view of the present to equip our troops combat readiness work equipment combat capability evaluation model is put forward, so that from the perspective of people, equipment, environment and so on to evaluate equipment readiness to work. At the same time, the index system of the combat readiness assessment model is determined, and the weight of each quantitative index is determined, and the dimensionless characteristic value of the qualitative index is fuzzy.

The combat readiness management of the troops is an important guarantee for the troops to have a higher level of combat readiness and combat effectiveness. The aim is to ensure that the equipment is in good technical and tactical condition. In order to effectively evaluate military equipment readiness work reasonably, promote the development of troops and equipment readiness work, build a reasonable equipment combat capability evaluation model, will be an important part of the equipment readiness to work.
\end{abstract}

\section{The Equipment Readiness Assessment Model is Proposed}

Military equipment combat readiness management is an important content of equipment management. In order to objectively evaluate the combat readiness management of the troops, the concept of equipment combat readiness and equipment readiness assessment model were put forward.

\section{Equipment combat readiness}

Combat readiness of military equipment is equipped with authorities at various levels, team for security forces carries out operations in the normal state of preparations, forces is an important component of equipment management work. Its purpose is in normal state, by organizing, planning, control, command, making combat readiness plan, plan scientifically, reasonable configuration, deployment, security forces, maximize reserve equipment and scientific management of war readiness material and strengthening the combat readiness mobilization, drills and combat readiness facilities construction, guarantee of military equipment is in good technical condition.

Military equipment combat readiness review is an important content and basic system of military equipment combat readiness management. In order to be truly test forces military equipment level, summarize constantly improve, effectively ensure the implementation of the combat readiness of military equipment, and it is necessary to build a unified index to measure the performance of equipment combat readiness work, thus puts forward the concept of equipment readiness capability. In this case, the ability to equip combat readiness is defined as the ability of the force to complete the specified equipment guarantee task without error in the specified time.

\section{Equipment combat readiness assessment model}

According to the human-computer environment system engineering theory, any working system that has been involved in it is called human-computer environment system. Equipment combat readiness management is a man-machine environment system, the "people" in the system are equipment management and use personnel; "Machine" is equipment; "Environment" is refers to the chosen people, equipment, coexistence of working conditions, including the internal environment of equipment itself, such as temperature, humidity, noise, vibration, etc., as well as the external operational environment of people and equipment, such as geography, climate, etc. In the process of analysis, research and evaluation of equipment combat readiness management, three factors of human, equipment and environment must be considered. But in early studies, the factors of 
equipment are often considered as priorities, and human factors are often overlooked. In fact, the operational mistakes of the combat-ready workers and the errors caused by environmental conditions are not negligible. During combat capability evaluation, therefore, need to scientifically from the three key elements of people, equipment, environment and their relations, comprehensive consideration, reasonable equipment combat capability evaluation model is set up. The mathematical formula of the equipment readiness assessment model is:

$$
E_{s}=f\left(E_{h}, E_{m}, E_{e}\right)
$$

Type: $E_{s}$ is equipment integrated combat capability, which is considering various factors in the operation of equipment readiness ability to influence and form integrated combat capability, $E_{h}$ is ability to combat readiness, a factor is equipped with comprehensive capability of combat readiness, $E_{m}$ is the combat ability of the equipment, the main means for the equipment itself in technical, quality and performance of the objective aspects of equipment comprehensive capability of combat

readiness, $E_{e}$ is the influence of environmental factors on the ability to combat readiness, the natural factors. Because of people's ability to combat readiness and equipment readiness capability is influenced by many factors, environmental factors at the same time itself is uncertain, so the equipment comprehensive combat ability is by a variety of operational conditions and joint decision of the internal environment of people and equipment. The following will further analyze and define the variables in the model.

\section{Determination of various elements in the model}

The elements of the model, equipment and environment are restricted and affected by various elements. To determine the elements, the various elements of each element must be listed. Since the influence of each element on the equipment combat readiness is different, it is generally not possible to use the equal weight, so it is necessary to determine their respective weights according to their degree of influence on the factor.

To determine the elements contained in each element, it can be calculated from the actual work of the equipment management, which will affect the various elements of people, equipment, environment and other elements. The methods of determining the weight of various elements are: Delphi method, expert investigation method, analytic hierarchy process, fuzzy analytic hierarchy process and various kinds of improved analytic hierarchy process.

\section{Determination of the Model Index System}

Affect the ability of the equipment readiness people, equipment, environmental factors, respectively is composed of different elements, only the comprehensive consideration of various elements on the impact of these factors, can more accurately determine each factor, thus more objectively evaluate the troops and equipment readiness work.

\section{Personnel elements}

There are many elements that influence people's elements, which can be divided into intrinsic factors and external factors. The inherent factors include: human's visual, hearing, touch, smell, thinking, judgment, decision-making ability, the structure of the human body, such as psychological quality and levels of knowledge, the external factors include: personnel, collaboration in liability system, incentives, supervision mechanism, etc. The effects on the staff.

In the process of evaluation, the baseline value of personnel ability should be determined first, and the base value should be determined on the basis of a large-scale field investigation and a comprehensive evaluation of the personnel factors in various aspects. According to the intrinsic factors of personnel, a variety of single-soldier assessment can be set specifically to detect the various abilities of the measured personnel. For the external factors of personnel, group assessment can be set up to find out the organization management and coordination ability of the force. At the same time, the knowledge level of personnel should be combined to make fuzzy comprehensive 
evaluation.

\section{Equipment elements}

The equipment factor has a great influence on the ability of combat readiness, and the readiness rate of the equipment and the availability of the equipment can directly reflect the availability of the equipment. Affect the ability of the equipment readiness elements include: equipment itself inherent properties, lighter and less equipment such as external properties. In the assessment, the technical and tactical performance of the equipment and the troop's management and arrangement of equipment should be taken into account, combining with the specific combat background, to properly assess the equipment readiness of a force.

\section{Environmental elements}

Environmental factors have a direct impact on operational capability, and different combat styles have different requirements for equipment combat readiness. Environmental elements include: humidity, temperature, vibration, electromagnetic radiation, light radiation, noise, thunderstorm, storm, pressure, dust, harmful gas, etc. In addition, the equipment management system and incentive mechanism of the army are also the environmental factors that influence the equipment readiness. When conducting an assessment, consider the potential battlefield environment and the ongoing military preparedness tasks. Combine these environment to the personnel, the influence that the equipment causes to carry on comprehensive consideration.

\section{The Standard Index of the Naval Equipment Readness Construction}

AHP method is the determination method of weight in analytic hierarchy process.

Set the factors $U=\left\{U_{1}, U_{2}, \cdots, U_{q}\right\}$, need to determine the weight vector $\mathbf{w}=\left(w_{1}, w_{2}, \cdots, w_{q}\right)^{T}$.

To target the index $U$, first construct the judgment matrix. The relative importance of the factors to the upper index is analyzed and determined by two or two comparisons of all factors in the factor set. The results of the judgment can be represented in the judgment matrix, as shown in table 3.1 .

Table 3.1 judgment matrix table

\begin{tabular}{|c|cccc|}
\hline$U$ & $U_{1}$ & $U_{2}$ & & $U_{q}$ \\
\hline$U_{1}$ & $a_{11}$ & $a_{12}$ & $\cdots$ & $a_{1 q}$ \\
$U_{2}$ & $a_{21}$ & $a_{22}$ & $\cdots$ & $a_{2 q}$ \\
$\vdots$ & $\vdots$ & $\vdots$ & $\cdots$ & $\vdots$ \\
$U_{q}$ & $a_{q 1}$ & $a_{q 2}$ & $\cdots$ & $a_{q q}$ \\
\hline
\end{tabular}

In table 3.1, the relative $U_{i}$ importance scale of the relative importance $U_{j}$ is expressed in terms of quantity.

$a_{i j}$ can be quantified according to actual experience, also can be obtained by following method.

From the two comparison of various factors in order to get the quantitative judgment matrix, according to the research on the laws of the people's psychological characteristic and thought, found that people distinguish between the limit of the level of information capacity is 9, thus put forward by 9 important level two comparative judgment, using 1 9 integers or from bottom to do quantitative processing.

According to the judgment matrix, the following eigenvalue problems are solved

$$
\mathbf{A} \mathbf{w}=\lambda_{\max } \mathbf{w}
$$

Among them: $\mathbf{A}=\left(a_{i j}\right)_{q \times q}$ is the judgment matrix, $\lambda_{\max }$ is the $\mathbf{A}$ maximum eigenvalue, $\mathbf{w}$ is the $\lambda_{\max }$ corresponding eigenvector. That's what we're looking for relative importance weight 
vectors. In other words, what we're looking for is the eigenvectors that correspond to the maximum eigenvalues of the matrix.

The following approximate method is used to describe the reasons for expressing the sorting weight with the feature vectors. If there is $q$ objects, their weight is respectively $w_{1}, w_{2}, \cdots, w_{q}$, $\sum_{i=1}^{q} w_{i}=1$. If you compare them to two, the ratio of them can be the order matrix $\mathbf{A}$.

$\mathbf{A}=\left(\begin{array}{cccc}\frac{w_{1}}{w_{1}} & \frac{w_{1}}{w_{2}} & \cdots & \frac{w_{1}}{w_{q}} \\ \frac{w_{2}}{w_{1}} & \frac{w_{2}}{w_{2}} & \cdots & \frac{w_{2}}{w_{q}} \\ \vdots & \vdots & \vdots & \vdots \\ \frac{w_{q}}{w_{1}} & \frac{w_{q}}{w_{2}} & \cdots & \frac{w_{q}}{w_{q}}\end{array}\right)$

If you use the weight vector $\mathbf{w}=\left(w_{1}, w_{2}, \cdots, w_{q}\right)^{T}$ So we get the right multiplication matrix:

$$
\mathbf{A w}=\left(\begin{array}{cccc}
\frac{w_{1}}{w_{1}} & \frac{w_{1}}{w_{2}} & \cdots & \frac{w_{1}}{w_{q}} \\
\frac{w_{2}}{w_{1}} & \frac{w_{2}}{w_{2}} & \cdots & \frac{w_{2}}{w_{q}} \\
\vdots & \vdots & \vdots & \vdots \\
\frac{w_{q}}{w_{1}} & \frac{w_{q}}{w_{2}} & \cdots & \frac{w_{q}}{w_{q}}
\end{array}\right)=q\left(\begin{array}{c}
w_{1} \\
w_{2} \\
\vdots \\
w_{q}
\end{array}\right)=q\left(\begin{array}{c}
w_{1} \\
w_{2} \\
\vdots \\
w_{q}
\end{array}\right)=q \mathbf{w}
$$

$(\mathbf{A}-q \mathbf{I}) \mathbf{w}=\mathbf{0}$

The equation (5) indicates that the eigenvector of the matrix $\mathbf{w}$ is the unique eigenvalue of the matrix A. If two or two comparisons of object weight are determined to determine the judgment matrix, then the equation (5) eigenvector can be solved to determine the relative weight of each object.

For the actual judgment matrix, each judgment cannot be as accurate as weighing, but we can assume that these judgments are judged by a small deviation of accurate judgment. According to prron-frobenius, this kind of positive and reciprocal judgment matrix has the only positive eigenvalue and corresponding positive eigenvector. Moreover, it can be proved that the calculated eigenvector $\mathrm{W}$ is only slightly different for the small perturbation of the judgment matrix. This shows that the use of eigenvectors as sort weights is not only reasonable, but also has good stability.

A consistency check is required for the constructed judgment matrix. Usually, if the element in the matrix is satisfied: $a_{i j}=a_{i k} / a_{j k}$ or $a_{i k}=a_{i j} a_{j k} \quad(i, j, k=1,2, \cdots, q)$, we call such a matrix a complete identity matrix. But because of the complexity of the objective things, the limitations of subject awareness and understanding between the main body of the diversity, often accompanied by error, so the judgment matrix is completely consistent judgment matrix impossible. AHP law requires, therefore, to make time order judgment matrix $q$ pairwise comparison, in order to export a comparatively reasonable reflect policymakers judge ordering, and the hard to avoid disturbance (error) with multiple judgment, can have the effect of each counter, bringing the total sorting result is isotonic. Of course, the error of each judgment should not be too large, and the whole judgment matrix should not deviate from the consistency too large, otherwise it will also affect the preorderability of the results. Through the theoretical research and social practice of saadi and his colleagues, the method of consistency test was summarized.

\section{Conclusion}

The equipment combat readiness management is an important part of the combat readiness management, and the ability of equipment combat readiness determines the size of equipment 
operation and guarantee capability. The ability to properly assess the equipment readiness of troops can improve the level of equipment management. In this paper, the author gives a preliminary consideration to the establishment of the equipment combat readiness assessment model, thus providing a quantifiable assessment method for the military equipment management level.

\section{References}

[1] Raymond A. Pyles, Robert S. Tripp. A Common Operating Picture for Air Force Materiel Sustainment [R]. www.rand.org. 2008.1-2

[2] Danid Keuhlen, Oliver L.Bryant, Kenneth K. Young. The Common Operational Picture in Joint Vision 2020: A Less Layered Cake[C]. Joint Forces Staff College Joint and Combined Warfare School, 2002.

[3] Ahuja G.2000. Collaboration networks,structural holes and innovation: A longitudinal study[J]. Administrative Science Quarterly, 45:425 455.

[4] Dr.David Baar, Garth Shoemaker. Pliable Display Technology for the Common Operational Picture[R]. IDELIX Software Inc. 